\title{
Inversion recovery zero echo time (IR-ZTE) imaging for direct myelin detection in human brain: a feasibility study
}

\author{
Hyungseok Jang ${ }^{1}$, Michael Carl ${ }^{2}$, Yajun $\mathrm{Ma}^{1}$, Adam C. Searleman ${ }^{1}$, Saeed Jerban ${ }^{1}$, Eric Y. Chang ${ }^{1,3}$, \\ Jody Corey-Bloom ${ }^{4}$, Jiang $\mathrm{Du}^{1}$ \\ ${ }^{1}$ Department of Radiology, University of California San Diego, San Diego, CA, USA; ${ }^{2}$ GE Healthcare, San Diego, CA, USA; ${ }^{3}$ Radiology Service, \\ Veterans Affairs San Diego Healthcare System, San Diego, CA, USA; ${ }^{4}$ Department of Neurosciences, University of California, San Diego, CA, USA \\ Correspondence to: Jiang Du, PhD. Department of Radiology, University of California, San Diego, 200 West Arbor Drive, San Diego, CA 92103-8226, \\ USA. Email: jiangdu@ucsd.edu.
}

Background: Myelin alteration is closely associated with neurological diseases such as multiple sclerosis (MS). Unfortunately, due to myelin's extremely short T2* ( 0.3 ms or shorter at 3T), it cannot be directly imaged with conventional MR imaging techniques. Recently, ultrashort echo time (UTE) imaging-based methods have been proposed for direct imaging of myelin. In this study, we explore the feasibility and efficacy of inversion recovery prepared zero echo time (IR-ZTE) imaging for direct volumetric imaging of myelin in white matter of the brain in vivo.

Methods: In the proposed method, an adiabatic IR preparation pulse is used to suppress long T2 white matter signal, followed by dual echo ZTE imaging where the remaining long T2 components, including gray matter, are suppressed by dual echo subtraction. In the implementation of ZTE, the sampling strategy introduced in Water- and Fat-Suppressed Proton Projection MRI (WASPI) was incorporated to acquire the k-space data missing due to the radiofrequency (RF) transmit/receiver switching time. The IR-ZTE sequence was implemented on a $3 \mathrm{~T}$ clinical MR system and evaluated using a myelin phantom composed of six different myelin concentrations ( $0 \%$ to $20 \%$ ), a cadaveric human brain, four healthy volunteers, and seven MS patients.

Results: In the myelin phantom experiment, the ZTE signal intensity showed high linearity to the myelin concentrations $\left(\mathrm{R}^{2}=0.98\right)$. In the ex vivo and in vivo experiments, the IR-ZTE sequence provided high contrast volumetric imaging of myelin in human brains. The IR-ZTE sequence was able to detect demyelinated foci lesions in all MS patients.

Conclusions: Adiabatic IR prepared dual echo ZTE imaging allows for direct, volumetric imaging of myelin in white matter of the brain in vivo.

Keywords: Inversion recovery (IR); myelin; multiple sclerosis (MS); ultrashort echo time (UTE); zero echo time (ZTE)

Submitted Dec 03, 2019. Accepted for publication Apr 01, 2020.

doi: $10.21037 /$ qims.2020.04.13

View this article at: http://dx.doi.org/10.21037/qims.2020.04.13

\section{Introduction}

Myelin is an essential component for neurotransmission in the central nervous system (CNS) and the peripheral nervous system (PNS), forming a water and lipid bilayer structure which wraps around each axon. Loss of myelin in the neural system can cause severe dysfunction in visual, motor, sensory, and autonomic processes (1). Myelin alteration is closely associated with neurological diseases such as multiple sclerosis (MS) (2), mild traumatic brain injury (3), central pontine myelinolysis (4), and acute disseminated encephalomyelitis (5). Therefore, 
the assessment of demyelination and remyelination can provide critical information for diagnosis and prognosis of neurological disorders. While histology is the gold standard method to assess myelination, it cannot be performed on human subjects. Thus, clinicians lack an established, non-invasive tool to provide direct information regarding myelination in patients.

MRI is one of the most capable imaging modalities available to provide qualitative and quantitative imaging of the brain. MR sequences such as T2-weighted fast spin echo or T2-weighted fluid-attenuated inversion recovery (FLAIR) have been used to provide contrast between gray matter, white matter, and fluid, a differentiation which reveals demyelinated lesions in patients $(6,7)$. Diffusion tensor imaging has been used to resolve the connectivity of axons in white matter of the brain $(6,8)$. Magnetization transfer has also been proposed to assess myelination in a brain $(6,9,10)$. Myelin water imaging has been investigated to estimate demyelination based on the information of water in myelin with short T2* (11-13). However, those methods are only capable of providing indirect information regarding myelin. Direct myelin imaging in MRI remains a challenge due to the extremely short $\mathrm{T} 2$ decay of myelin ( $\mathrm{T}^{*} \sim 0.3 \mathrm{~ms}$ or shorter at 3T) (14-17). Recently, inversion recovery (IR)-prepared ultrashort echo time (UTE) imaging has emerged as an effective imaging method to resolve the myelin signal with improved dynamic range $(16,18-22)$. In this method, an adiabatic inversion pulse is utilized to uniformly invert the longitudinal magnetizations of long T2 water components with little sensitivity to B1 inhomogeneity. At the nulling point of long T2 components in white matter, a dual echo UTE imaging is performed, and the myelin image is obtained by echo subtraction.

Myelin has a broad spectrum, and there may be multiple components with different T2*s $(16,17)$. Therefore, the acquired myelin signal may differ according to the sampling strategy parameters, including readout trajectory and duration. Multiple imaging techniques have been proposed for UTE imaging: single point imaging (SPI) $(23,24)$; Sweep Imaging with Fourier Transform (SWIFT) (25-27); zero echo time (ZTE) imaging, which shortens echo time (TE) by using short radiofrequency (RF) pulse and constant readout gradient (28-32); Pointwise Encoding Time Reduction With Radial Acquisition (PETRA), which is similar to ZTE but which utilizes SPI encoding to fill the missing data during RF coil deadtime (33); ramped hybrid encoding (RHE), which improves PETRA to minimize readout duration (34-36); variable TE (VTE) imaging, which benefits from Cartesian sampling pattern with shortened variable TE $(37,38)$; and UTE-Cones imaging, which allows efficient sampling using 3D spiral encoding (39-43). In our previous work, we showed that it was feasible to achieve direct myelin imaging with IR-prepared Cones-UTE and RHE techniques (44-46).

ZTE is a promising UTE technique for direct myelin imaging due to its constant sampling using a pre-ramped constant gradient, whereby the short $\mathrm{T} 2{ }^{*}$ components in myelin can be captured more efficiently. However, ZTE also has limitations in applicable flip angle (FA) and readout bandwidth ( $\mathrm{rBW}$ ) due to the application of RF pulse with the constant readout gradient turned on, which, if not appropriately tuned, can cause loss of central k-space and unwanted signal drop-off in the periphery of the field of view (FOV). In this study, we evaluate the feasibility of dual echo IR-ZTE for volumetric, qualitative myelin imaging using a myelin phantom and a cadaveric human brain specimen, as well as healthy volunteers and MS patients.

\section{Methods}

\section{Adiabatic IR preparation}

Myelin has an extremely short $\mathrm{T} 2^{*}(\sim 0.3 \mathrm{~ms}$ or shorter at $3 \mathrm{~T})$ and a low proton density, and therefore shows a much lower signal intensity than other long T2 tissues or tissue components, even when using UTE MRI $(14,15)$. The low myelin signal is prone to imaging artifacts and signal bias caused by strong signal from surrounding long T2 components (e.g., white matter, gray matter, and CSF). In this study, adiabatic IR was utilized to suppress long T2 white matter and to improve myelin contrast with reduced artifacts and improved dynamic range. Figure $1 \mathrm{~A}$ shows an example of typical IR after application of an adiabatic inversion pulse. Due to the long pulse width of the adiabatic inversion pulse $(\sim 9 \mathrm{~ms})$ and extremely short $\mathrm{T}^{*}$ decay of myelin ( $\sim .3 \mathrm{~ms}$ or shorter), the magnetization of myelin is not inverted but partially saturated. Other long T2 components including white matter and gray matter are evenly inverted by the adiabatic inversion pulse. By performing ZTE imaging at the inversion time (TI) tuned at the nulling point of white matter water signal, the long T2 white matter signal can be suppressed, as shown in Figure $1 B$. Note that multiple spokes are acquired per IR preparation for time-efficient $3 \mathrm{D}$ imaging. The remaining long T2 signals are further suppressed by dual echo subtraction. 
A

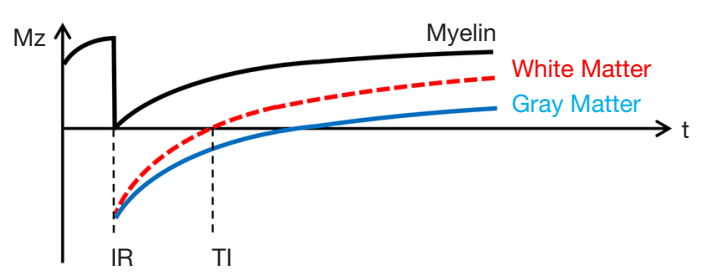

C

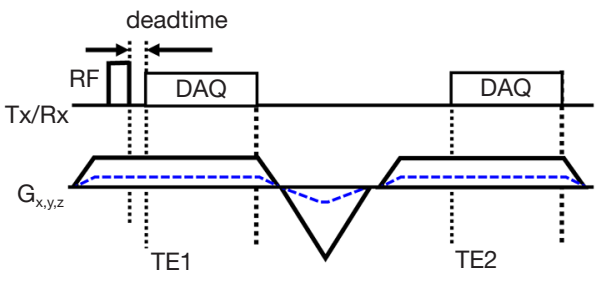

B

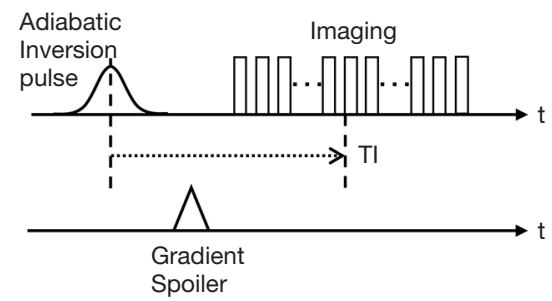

D

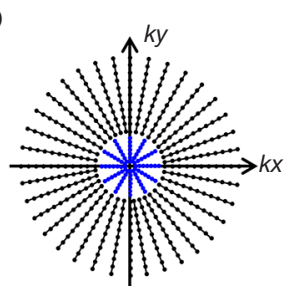

Figure 1 Pulse sequence diagram. (A) Typical adiabatic inversion recovery signal changes for white matter, gray matter, and myelin in the brain; (B) adiabatic inversion prepared multi-spoke imaging; (C) dual echo ZTE imaging; and (D) a 2D example of sampling patterns in ZTE with WASPI encoding. WASPI is used to fill the hole: the missing data is acquired using reduced readout bandwidth, as depicted by the dotted blue line in (C). A smaller number of spokes is typically required to fill the hole, as illustrated in (D). ZTE, zero echo time; WASPI, Water- and Fat-Suppressed Proton Projection MRI.

\section{Dual echo IR-ZTE}

Figure $1 C$ shows the pulse sequence diagram of the dual echo ZTE. In this acquisition scheme, ZTE (TE1) is followed by gradient recalled echo (TE2). ZTE is often characterized as radial center-out readout using constant gradient applied before RF pulse. This is a fundamental difference from conventional UTE imaging techniques where readout gradients are applied after the RF pulse. Due to the use of the plateau of the gradient, which is already ramped up before RF excitation, ZTE allows capture of the shortly decaying signal, which conventional sequences may miss in ramping up the readout gradient. On the other hand, during the RF transmit/receiver switching time, which is a blind time for data readout, the signal is not acquired, leaving a hole in the k-space center. In this study, the sampling strategy introduced in Water- and FatSuppressed Proton Projection MRI (WASPI) (47) is used to fill the hole, and the missing data is acquired using reduced rBW, as depicted by the dotted blue line in Figure 1C. A smaller number of spokes is typically required to fill the hole as illustrated in Figure 1D. The reduction rate of rBW is commonly referred to as the WASPI factor.

\section{Experimental setup}

The IR-ZTE sequence was implemented on a clinical $3 \mathrm{~T}$
MR system (MR750, GE Healthcare, Waukesha, WI). A GE-provided adiabatic inversion pulse (Silver Hoult, pulse width of $8.64 \mathrm{~ms}$, bandwidth of $1.5 \mathrm{kHz}$ ) was used. To evaluate the IR-ZTE sequence in myelin imaging in human brains, imaging experiments with a myelin phantom, a cadaveric human brain (56-year-old female donor), four healthy volunteers (males aged $34.5 \pm 2.3$ ), and seven MS patients (females aged $53.7 \pm 13.9$ ) were performed. All human subjects were recruited in accordance with the institutional review board. Signed consent was collected prior to the scan. For the phantom experiment, a $\mathrm{D}_{2} \mathrm{O}$ myelin phantom was prepared by compounding myelin lipid powder (type 1 bovine brain lipid extract, Sigma-Aldrich $\mathrm{B} 1502$, St. Louis, $\mathrm{MO}$ ) with distilled, deionized $\mathrm{D}_{2} \mathrm{O}$ in $1.0-\mathrm{mL}$ syringes (diameter of $\sim 4.7 \mathrm{~mm}$ ). The phantom was comprised of six tubes of six different myelin concentrations: $0,4 \%, 8 \%, 12 \%, 16 \%$, and $20 \%$ weight/volume (grams of solute per $1 \mathrm{~mL}$ of solution) as shown in Figure $2 \mathrm{~A}$. The six tubes were bundled together with tape and inserted into a single, empty $30-\mathrm{mL}$ syringe (diameter of $\sim 22.9 \mathrm{~mm}$ ).

\section{Imaging parameters}

All scans were performed at 3T (MR750). The $\mathrm{D}_{2} \mathrm{O}$-myelin phantom was scanned using a custom-made transmit/ receive birdcage RF coil (single channel) with the following 

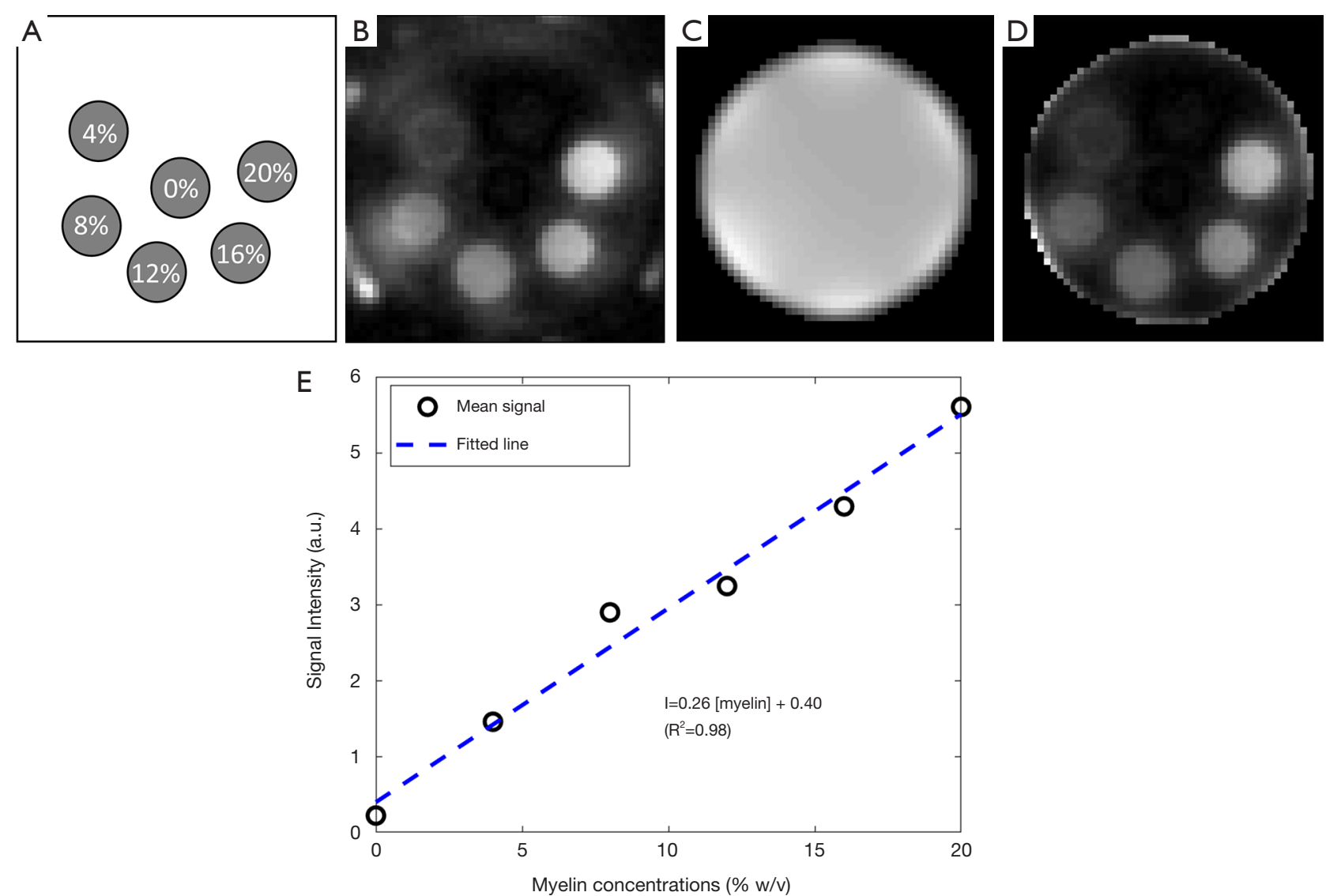

Figure 2 Myelin phantom experiment. (A) Tubes with different myelin concentrations ranging from 0-20\%; (B) a representative ZTE image at TE $=12 \mu \mathrm{s}$; (C) the corresponding coil sensitivity map; (D) the ZTE image after coil sensitivity correction; and (E) the scatter plot and fitted curve showing mean ZTE signal intensity versus myelin concentration. The ZTE signal intensity shows high linearity to the myelin concentrations. ZTE, zero echo time; TE, echo time.

imaging parameters: non-IR single echo ZTE imaging, TR $=20 \mathrm{~ms}, \mathrm{TE}=28 \mu \mathrm{s}, \mathrm{FA}=6^{\circ}$ with pulse width $=20 \mu \mathrm{s}$, FOV $=50 \times 50 \times 120 \mathrm{~mm}^{3}$, matrix size $=96 \times 96 \times 40, \mathrm{rBW}=62.5 \mathrm{kHz}$, WASPI factor $=8$, \# of WASPI encoding $=2160$, \# of radial frequency encoding $=27360$, and scan time $=10 \min 4 \mathrm{~s}$. To acquire a coil sensitivity map, the imaging was repeated with a syringe filled with saline.

For ex vivo brain imaging, the scan was performed with a 12-channel receive-only head coil (GE Healthcare) using the following imaging parameters: an adiabatic inversion pulse applied, TR $=1,000 \mathrm{~ms}$, TI $=310 \mathrm{~ms}, \mathrm{TE}=12 \mu \mathrm{s} / 3.5 \mathrm{~ms}$, Tau (timing between RF pulses) $=10.6 \mathrm{~ms}, \mathrm{FA}=6^{\circ}$ with pulse width $=20 \mu \mathrm{s}, \mathrm{FOV}=220 \times 220 \times 160 \mathrm{~mm}^{3}$, matrix size $=200 \times 200 \times 40, \mathrm{rBW}=62.5 \mathrm{kHz}$, WASPI factor $=8$, \# of WASPI encoding $=544$, \# of radial frequency encoding $=33856$, \# of spokes per IR preparation $=16$, inter-spoke TR $=10 \mathrm{~ms}$, and scan time $=35 \mathrm{~min} 54 \mathrm{~s}$.
For in vivo brain imaging, the scan was performed with a 12-channel receive-only head coil (GE Healthcare) using the following imaging parameters: (I) magnetization prepared rapid acquisition with gradient echo (MPRAGE) (48): $\mathrm{FA}=12^{\circ}, \mathrm{FOV}=256 \times 256 \times 178 \mathrm{~mm}^{3}$, matrix size $=256 \times 256 \times 148, \mathrm{rBW}=83.4 \mathrm{kHz}, \mathrm{TE}=3.2 \mathrm{~ms}$, TR/ $\mathrm{TI}=8.2 / 450 \mathrm{~ms}$, acceleration factor $=4$, scan time $=5 \mathrm{~min}$. (II) FLAIR: $\mathrm{FA}=90^{\circ}, \mathrm{FOV}=256 \times 256 \times 256 \mathrm{~mm}^{3}$, matrix size $=256 \times 256 \times 256, \mathrm{rBW}=83.4 \mathrm{kHz}, \mathrm{TE}=116.5 \mathrm{~ms}$, TR/ $\mathrm{TI}=7,600 \mathrm{~ms} / 2,162 \mathrm{~ms}$, acceleration factor $=4$, scan time $=6 \mathrm{~min} 54 \mathrm{~s}$. (III) IR-ZTE: an adiabatic inversion pulse applied, TR $=1,000 \mathrm{~ms}$, TI $=340 \mathrm{~ms}$, Tau $=8.44 \mathrm{~ms}$, TE $=12 \mu \mathrm{s} / 2.5 \mathrm{~ms}, \mathrm{FA}=6^{\circ}$ with pulse width $=20 \mu \mathrm{s}$, FOV $=220 \times 220 \times 144 \mathrm{~mm}^{3}$, matrix size $=190 \times 190 \times 40, \mathrm{rBW}$ $=62.5 \mathrm{kHz}$, \# of WASPI encoding $=540$, \# of radial frequency encoding $=17100$, \# of spokes per IR preparation $=30$, and scan time $=9 \min 52 \mathrm{~s}$. 


\section{Data processing}

All MR images were reconstructed using online reconstruction code on the scanner host $\mathrm{PC}$, which was made using GE Orchestra SDK v1.7.1 to perform griddingbased image reconstruction. The following gridding parameters were used: alpha $=2$ and kernel width $=3$ data points. Density function was analytically calculated based on the inter-spoke distance and intra-spoke sampling density. The low-resolution k-space data acquired using WASPI were combined with high-resolution data using a linear merging filter with width of 2 data points. To reduce noise, the gridded $\mathrm{k}$-space was applied with a fermi filter with radius of 95 data points and width of 10 data points. For ex vivo and in vivo imaging, where a 12-channel head coil was used, the reconstructed images in each RF receiver channel were combined using the weighted sum of squares method, in which the weighting factors were calculated based on the noise power in each channel.

The myelin image was generated by applying magnitude echo subtraction. For the myelin phantom experiment, Matlab 2017b (The Mathworks Inc, Natick, MA, USA) was used to post-process the ZTE images and to perform curve fitting based on linear least square fitting. First, a coil sensitivity image was obtained by low-pass filtering (3D Gaussian filter with standard deviation of 1 pixel) a water phantom image acquired with ZTE. Then, the targeted myelin image acquired with ZTE was divided by the coil sensitivity map to correct for the variation in signal intensity due to the receiver coil sensitivity. ROIs for tubes were drawn manually well within the margins of the wall of the syringe to avoid pixels corrupted by MR signal from the tube itself.

\section{Results}

\section{Myelin phantom}

Figure $2 \mathrm{~B}$ shows the $\mathrm{D}_{2} \mathrm{O}$-myelin phantom image acquired with ZTE (at TE $=12 \mu \mathrm{s}$ ). Figure $2 C, D$ shows the acquired coil sensitivity map and the resultant ZTE image after correction for the coil sensitivity. Figure $2 E$ shows the mean signal in each tube and the fitted linear curve. The mean and standard deviation of the ZTE signal intensity was $0.22 \pm 0.11,1.46 \pm 0.09,2.90 \pm 0.17,3.25 \pm 0.10$, $4.30 \pm 0.20$, and $5.61 \pm 0.24$ for the tube with $0 \%, 4 \%, 8 \%$, $12 \%, 16 \%$, and $20 \%$ myelin concentrations, respectively. The mean signal intensity shows a strong linear relationship $\left(\mathrm{R}^{2}=0.98\right)$ with the corresponding myelin concentrations, with a slope of 0.26 and y-intercept of 0.40 .

\section{Ex vivo cadaveric buman brain}

Figure 3 shows the IR-ZTE image obtained with the ex vivo cadaveric human brain specimen. The frontal region of the head was filled with gas generated by natural decomposition of tissues. In the ZTE image (top), myelin signal is visible, but with poor contrast due to high signals from the surrounding gray matter. In the second echo image (middle), the myelin signal decays to near-zero, which confirms that there is no remaining white matter water signal in the ZTE image. What we observe, then, is nearly pure short T2 component, which includes myelin lipid. In the subtraction image (bottom), the myelin is detected with high contrast, with suppression of the surrounding long $\mathrm{T} 2 *$ tissues.

\section{In vivo buman brain}

For all four healthy volunteers, IR-ZTE achieved high contrast direct myelin imaging. Figure 4 shows the in vivo result of a representative healthy volunteer (36-year-old male), which shows high contrast for myelin in the echosubtracted images. Slice selectivity artifacts are visible in the frontal area of head, as indicated by yellow arrows, but they did not significantly affect the brain region. For all seven MS patients, IR-ZTE resolved myelin signal in the echo subtraction image. Note that low-frequency signal bias is visible in the region indicated by a red arrow in the echosubtracted image, which slightly degrades myelin contrast.

Figure 5 shows results from two representative MS patients (Figure 5A: 73-year-old female, Figure 5B: 40-year-old female, Figure 5C: 38-year-old female), including T2-weighted FLAIR, T1-weighted MP-RAGE, and the proposed IRZTE-based myelin images. Demyelinated foci lesions are shown as bright and dark spots in FLAIR and MP-RAGE images, respectively. As the yellow arrows indicate, the foci lesions are also well detected by IR-ZTE. IR-ZTE sequence provides myelin-specific, morphological information of the foci lesions.

\section{Discussion}

In this study, we showed that IR-ZTE is feasible for direct volumetric myelin imaging. In the myelin phantom experiment, ZTE signal showed high linearity to myelin concentrations. The result implies that ZTE encoding scheme allows direct imaging of myelin. By calibrating the 


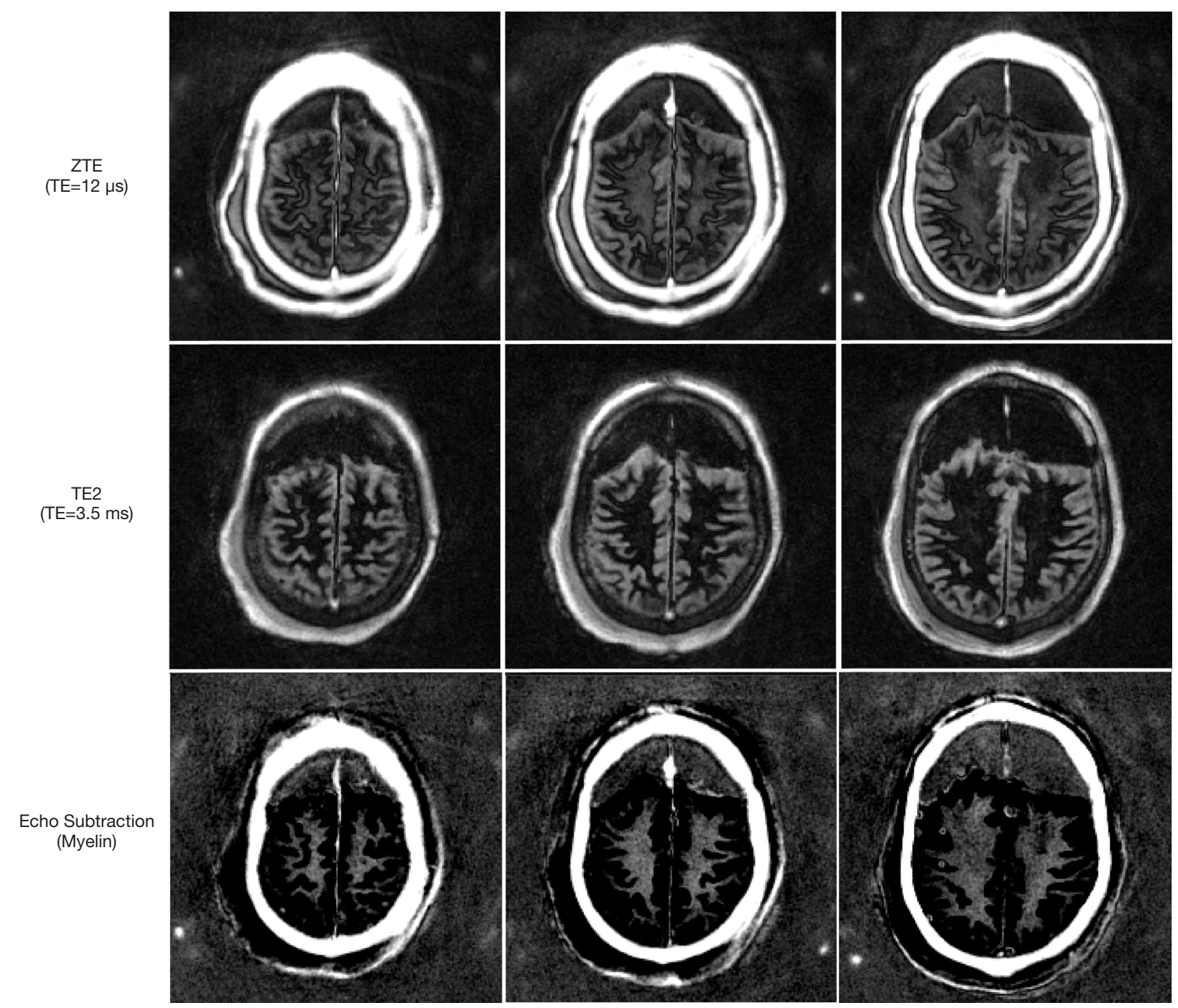

Figure 3 Ex vivo experiment with a cadaveric human brain (56-year-old female donor). In the ZTE image (top), myelin signal is visible, but with poor contrast due to high signal from the surrounding gray matter. In the second echo image (middle), the myelin signal decays to near-zero, which confirms that there is no remaining white matter water signal in the ZTE image; therefore, what we observe is nearly pure myelin lipid. In the echo subtraction image (bottom), the myelin is detected with high contrast and with suppression of the surrounding tissues. ZTE, zero echo time.

IR-ZTE signal to a reference phantom with known proton density, it was possible to map myelin proton density (after $\mathrm{T} 1$ and $\mathrm{T} 2 *$ correction, as the reference phantom and myelin may have different MR relaxation times). In the in vivo experiments with MS patients, IR-ZTE showed excellent detection of demyelinated foci lesions compared with conventional clinical sequences (e.g., MP-RAGE and FLAIR). Both MP-RAGE and FLAIR seemed to show ambiguous and underestimated MS lesions in regions indicated by yellow arrows in Figure 5, probably because the limited contrast in those sequences indirectly reflected demyelinated lesions.

It is promising that IR-ZTE is capable of resolving myelin signal in spite of the low rBW and FA that are commonly used to avoid signal drop-off in the periphery of FOV due to the unwanted and uncontrolled slice selectivity (34). To the best of our knowledge, this is the first study showing dual echo IR-ZTE for direct myelin imaging in human brains, although there are several animal and phantom studies reported in the literature $(16,22)$. Unlike in small 


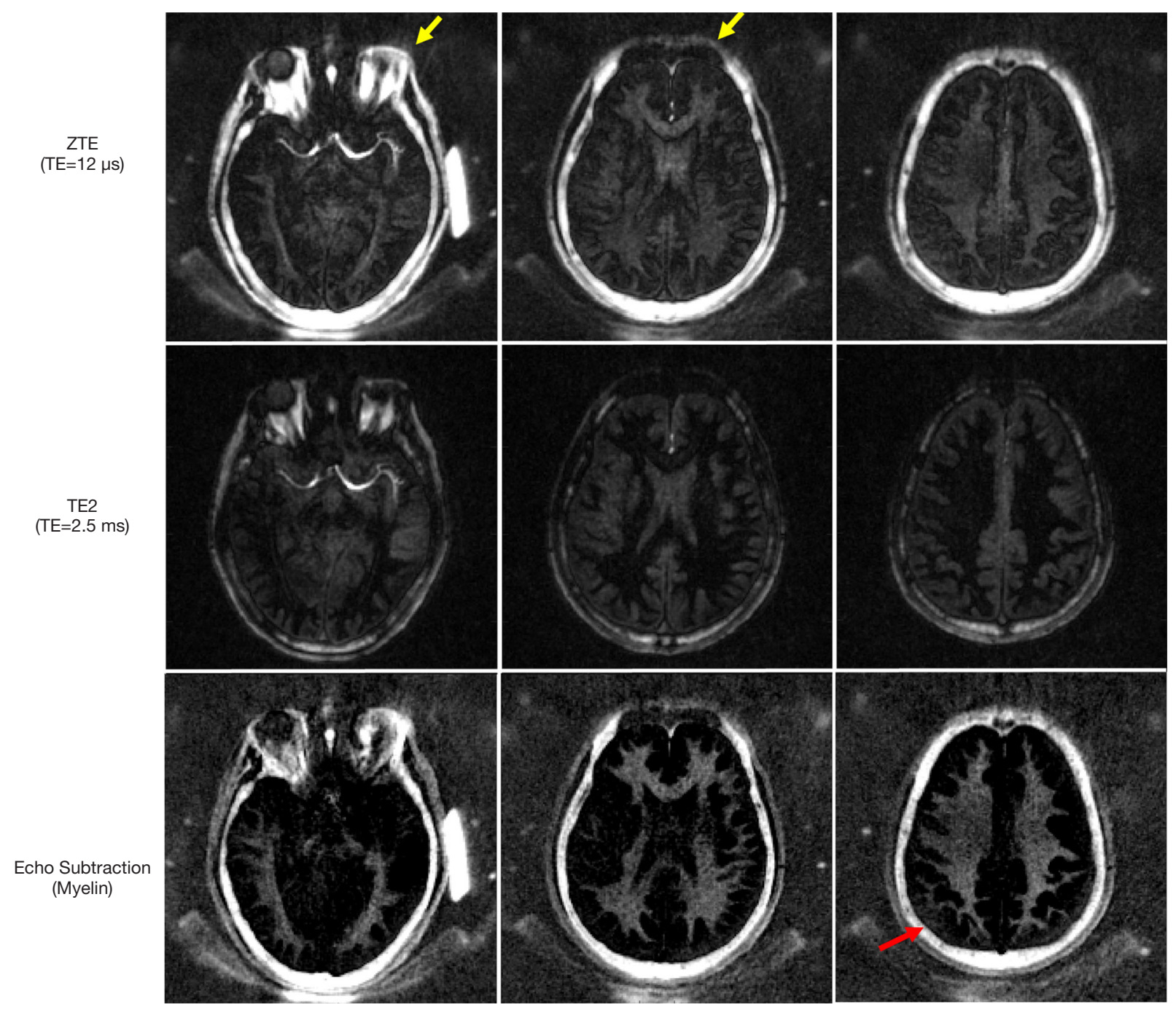

Figure 4 A healthy volunteer (36-year-old male). High contrast direct myelin imaging was achieved using the proposed dual echo IR-ZTE method. Subtraction of the ZTE image (top) from the second echo image (middle) provides high contrast images of myelin (bottom) in the brain of this volunteer, demonstrating the clinical feasibility of volumetric myelin mapping using the 3D dual echo IR-ZTE sequence. Slice selectivity artifact is shown in the images as indicated by yellow arrows. Low-frequency bias artifact is exhibited in the region indicated by a red arrow, which slightly degrades the myelin contrast. IR-ZTE, inversion recovery prepared zero echo time.

animals, where a small FOV is appropriate for imaging and which gives more flexibility in the choice of rBW and FA, human brain imaging is more difficult due to the larger FOV required to avoid imaging artifacts. In the current imaging protocol, a FA of $6^{\circ}$ was used with a $\mathrm{rBW}$ of $62.5 \mathrm{kHz}$ to avoid the slice selectivity artifact, which may have limited attainable signal-to-noise ratio. The slice selectivity artifact can be alleviated in the reconstruction process by identifying the pixel location with respect to the logical gradient isocenter and RF excitation profile $(49,50)$. Our future work will optimize the imaging parameters in IR-ZTE with slice selectivity correction to allow for higher FA.

As shown in Figure 4, low-frequency signal bias was observed in the echo subtracted image in IR-ZTE. Presumably, this is due to the imperfect signal acquisition in the missing central region in WASPI encoding. There is an inevitable difference in the encoding delay between the two encodings (i.e., high rBW encoding and low rBW WASPI encoding), which causes an abrupt change in signal intensity at the boundary due to the different $\mathrm{T} 2{ }^{*}$ decay. 
FLAIR

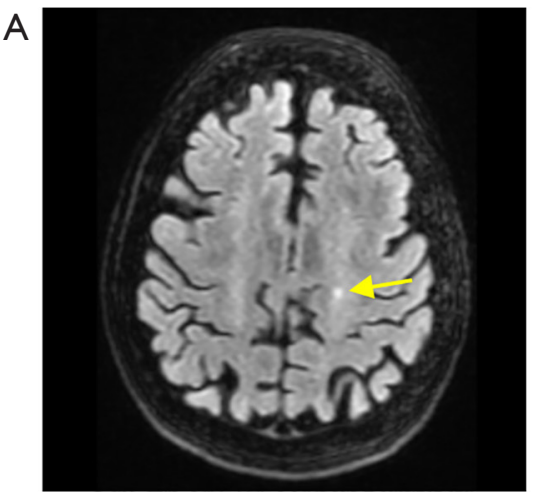

B

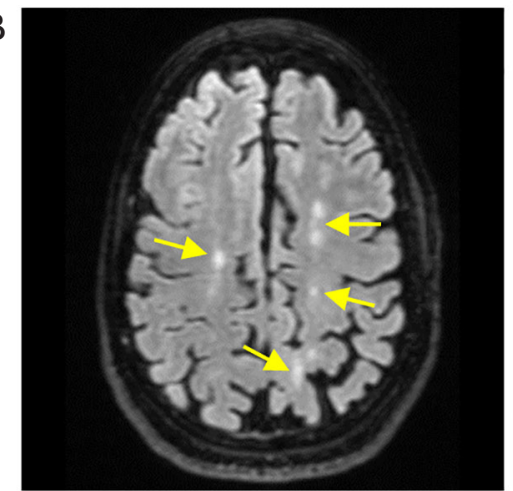

C

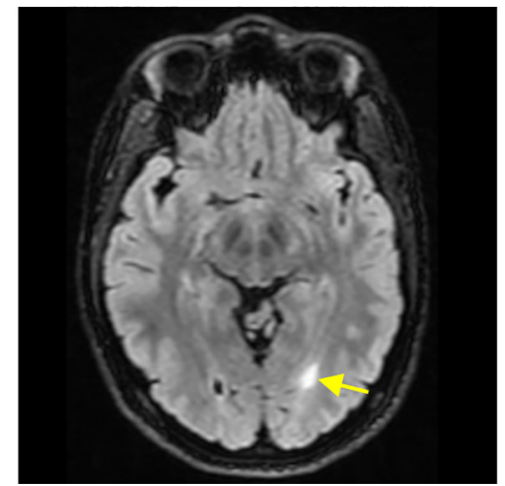

MP-RAGE
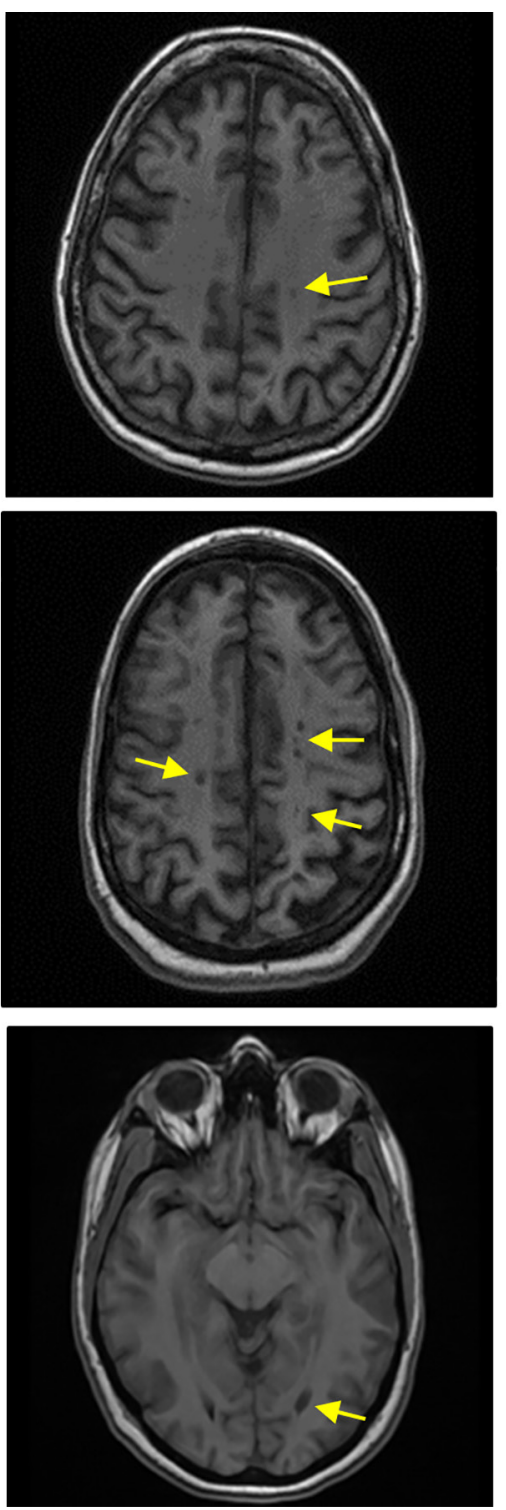

IR-ZTE
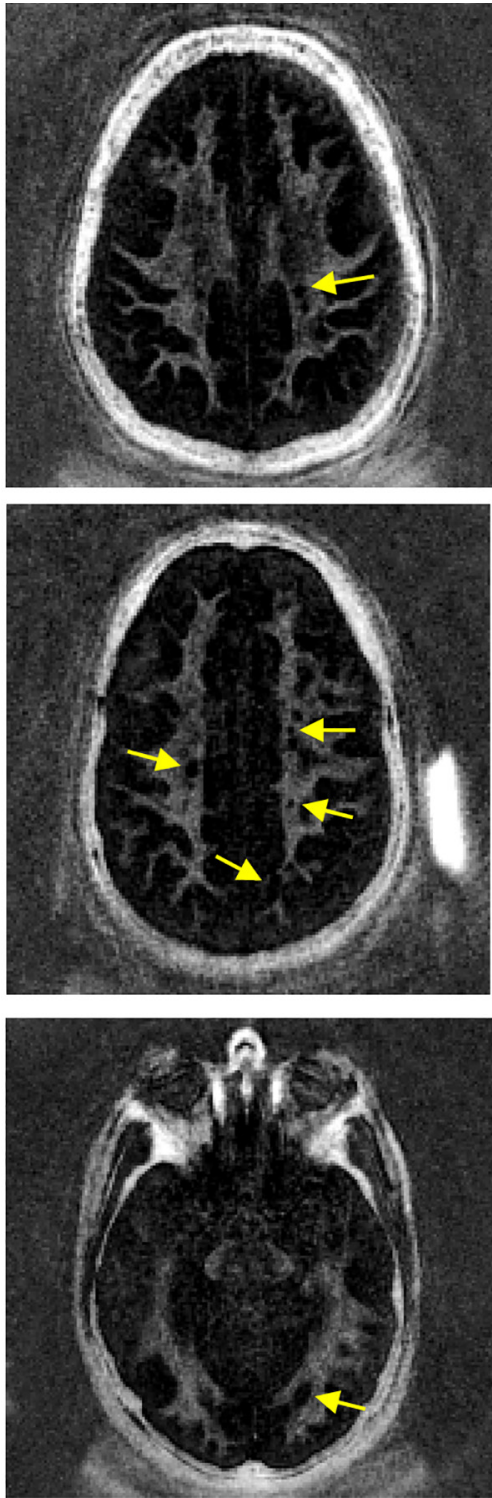

Figure 5 MS patients. (A) 73-year-old female; (B) 40-year-old female; and (C) 38-year-old female. The demyelinated foci lesions are shown as bright and dark spots in FLAIR and MP-RAGE images, respectively. As the yellow arrows indicate, the foci lesions are also well detected by IR-ZTE. MS, multiple sclerosis; FLAIR, fluid-attenuated inversion recovery; MP-RAGE, magnetization prepared rapid acquisition with gradient echo; IR-ZTE, inversion recovery prepared zero echo time.

Though we applied a merging filter to alleviate this effect, it is still very difficult to remove this artifact entirely. As the detected myelin signal is very low compared to the imaging artifacts, this artifact is crucial for myelin imaging. It will be interesting to investigate how this type of artifact is affected by different hole-filling strategies such as SPI or algebraic reconstruction. In future works, we will explore these different approaches in IR-ZTEbased myelin imaging $(51,52)$.

Dual-echo IR-UTE acquisition and echo subtraction has been used for selective imaging of myelin by several groups. Waldman et al. first reported this technique for myelin imaging in vivo at $1.5 \mathrm{~T}$ (21). Later Du et al. reported the use of 2D IR-UTE imaging of myelin in vivo at 3T, and 
reported a short $\mathrm{T} 2^{*}$ of $\sim 370 \pm 15 \mu$ s (53). The same group conducted a series of studies on myelin powders, myelin$\mathrm{D}_{2} \mathrm{O}$ paste, brain specimens, healthy volunteers and $\mathrm{MS}$ patients to further evaluate the capability of direct imaging of myelin in vivo on a clinical 3T scanner (14,15,19-21). However, their reported T2*s seem significantly longer than those reported by Horch et al. (17) and Wilhelm et al. (16). Wilhelm et al. investigated IR-UTE imaging and NMR spectroscopy of fresh rat spinal cord before and after $\mathrm{D}_{2} \mathrm{O}$ exchange, and demonstrated the capability of direct IR-UTE imaging of myelin at 9.4T. However, the IR-UTE dual-echo subtraction image likely included significant signal contamination from residual water as evidenced by the relative high signal in white matter in the second echo image with a TE of $1.2 \mathrm{~ms}$ (myelin signal should have decayed to near noise level at this TE). More recently, Weiger et al. employed two ZTE acquisitions with TEs of 15 and $503 \mu \mathrm{s}$, respectively (54). Subtraction of those two datasets was used to suppress long T2 signals and to create high contrast for myelin. However, this approach is expected to suffer from limited signal dynamic range and water contamination. As signal from water is far higher than signal from myelin, it is technically challenging to achieve selective myelin imaging through simple echo subtraction without other efficient long T2 suppression techniques. Clearly more research is needed to understand the signal source in UTE and ZTE imaging, especially dual-echo subtraction based techniques without or with long T2 suppression, of white matter and gray matter in the brain.

ZTE is commonly thought of as a silent sequence since it allows continuous and smooth ramp-up and ramp-down of the readout gradient during scanning. The current implementation of IR-ZTE does not allow this silent imaging feature due to the use of dual echo imaging, which requires gradient blips in different polarities within a TR. There is an alternative approach to implement the dual echo ZTE based on bipolar readout gradients. In this method, rewinding (or fly-back) gradient is not separately applied, but instead combined with the second readout gradient with opposite polarity (55). If only one spoke is acquired per IR preparation, it is feasible to use the bipolar readout scheme. However, if multiple spokes are acquired per IR preparation, which is essential in $3 \mathrm{D}$ imaging to save scan time, this can be problematic. Since each spoke is acquired at a slightly different TI in one set of IR, the acquired k-space is inevitably modulated by certain inter-spoke signal variations. If the bipolar readout scheme is used, the signal modulation in the encoded k-space will be different between the UTE and the second echo, which can cause signal bias artifacts in the echo subtraction. Moreover, since rBW is commonly set low in ZTE imaging, the use of bipolar gradient can be less time-efficient than using a separate rewinder gradient (to fly-back to the center of k-space), which is not limited by rBW.

ZTE is known to be advantageous in imaging extremely short $\mathrm{T}^{*}$ species such as myelin owing to the application of constant gradient, which allows very fast encoding immediately after RF excitation. In conventional UTE imaging schemes, the readout gradients are applied after RF deadtime, which requires additional time to ramp up the gradient to achieve the fastest encoding speed. Therefore, the effective TE in the encoded $\mathrm{k}$-space can be significantly longer than ZTE. However, ZTE is limited in the rBW. Those two competing factors make it difficult to conclude which encoding scheme is better for direct myelin imaging. In future works, different encoding schemes including ZTE, PETRA, RHE, SPI, and UTE-Cones will be compared in myelin phantoms, ex vivo samples, and in vivo human subjects to investigate the best imaging strategies for direct myelin imaging.

\section{Conclusions}

In this study, we demonstrated the feasibility of adiabatic IR prepared dual echo zero echo time imaging for direct, volumetric imaging of myelin in white matter of the brain in vivo.

\section{Acknowledgments}

Funding: The authors acknowledge grant support from the NIH (1R01 NS092650 and T32 EB005970), VA Clinical Science and Rehabilitation Research and Development Services (Merit Awards I01CX001388 and I01RX002604), UCSD Health Sciences Research Grant (RG096471), and GE Healthcare.

\section{Footnote}

Conflicts of Interest: All authors have completed the ICMJE uniform disclosure form (available at http://dx.doi. org/10.21037/qims.2020.04.13). MC reports other from GE Healthcare, during the conduct of the study. JD serves as an unpaid editorial board member of Quantitative Imaging in Medicine and Surgery. The other authors have no conflicts of interest to declare. 
Ethical Statement: The study was approved by institutional review board of University of California, San Diego (No. 151623). Written informed consent was obtained from the patient for publication of this study and any accompanying images.

Open Access Statement: This is an Open Access article distributed in accordance with the Creative Commons Attribution-NonCommercial-NoDerivs 4.0 International License (CC BY-NC-ND 4.0), which permits the noncommercial replication and distribution of the article with the strict proviso that no changes or edits are made and the original work is properly cited (including links to both the formal publication through the relevant DOI and the license). See: https://creativecommons.org/licenses/by-nc-nd/4.0/.

\section{References}

1. Polman CH, Reingold SC, Banwell B, Clanet M, Cohen JA, Filippi M, Fujihara K, Havrdova E, Hutchinson M, Kappos L, Lublin FD, Montalban X, O'Connor P, Sandberg-Wollheim M, Thompson AJ, Waubant E, Weinshenker B, Wolinsky JS. Diagnostic criteria for multiple sclerosis: 2010 Revisions to the McDonald criteria. Ann Neurol 2011;69:292-302.

2. Noseworthy JH, Lucchinetti C, Rodriguez M, Weinshenker BG. Multiple Sclerosis. N Engl J Med 2000;343:938-52.

3. Bigler ED, Maxwell WL. Neuropathology of mild traumatic brain injury: relationship to neuroimaging findings. Brain Imaging Behav 2012;6:108-36.

4. Gerber O. Central Pontine Myelinolysis. Arch Neurol 1983;40:116.

5. Garg RK. Acute disseminated encephalomyelitis. Postgrad Med J 2003;79:11-7.

6. Filippi M, Rocca MA. MR Imaging of Multiple Sclerosis. Radiology 2011;259:659-81.

7. Traboulsee A, Simon JH, Stone L, Fisher E, Jones DE, Malhotra A, Newsome SD, Oh J, Reich DS, Richert N, Rammohan K, Khan O, Radue EW, Ford C, Halper J, Li D. Revised Recommendations of the Consortium of MS Centers Task Force for a Standardized MRI Protocol and Clinical Guidelines for the Diagnosis and Follow-Up of Multiple Sclerosis. Am J Neuroradiol 2016;37:394-401.

8. Poonawalla AH, Hasan KM, Gupta RK, Ahn CW, Nelson F, Wolinsky JS, Narayana PA. Diffusion-tensor MR imaging of cortical lesions in multiple sclerosis: initial findings. Radiology 2008;246:880-6.
9. Wei H, Cao P, Bischof A, Henry RG, Larson PEZ, Liu C. MRI gradient-echo phase contrast of the brain at ultrashort TE with off-resonance saturation. Neuroimage 2018;175:1-11.

10. Alonso-Ortiz E, Levesque IR, Pike GB. MRI-based myelin water imaging: A technical review. Magn Reson Med 2015;73:70-81.

11. Deoni SC, Rutt BK, Arun T, Pierpaoli C, Jones DK. Gleaning multicomponent $\mathrm{T} 1$ and $\mathrm{T} 2$ information from steady-state imaging data. Magn Reson Med 2008;60:1372-87.

12. Lancaster JL, Andrews T, Hardies LJ, Dodd S, Fox PT. Three-pool model of white matter. J Magn Reson Imaging 2003;17:1-10.

13. Deoni SCL, Dean DC, O'Muircheartaigh J, Dirks H, Jerskey BA. Investigating white matter development in infancy and early childhood using myelin water faction and relaxation time mapping. Neuroimage 2012;63:1038-53.

14. Fan SJ, Ma Y, Chang EY, Bydder GM, Du J. Inversion recovery ultrashort echo time imaging of ultrashort $\mathrm{T} 2$ tissue components in ovine brain at $3 \mathrm{~T}$ : a sequential $\mathrm{D} 2$ O exchange study. NMR Biomed 2017;30:e3767.

15. Fan SJ, Ma Y, Zhu Y, Searleman A, Szeverenyi NM, Bydder GM, Du J. Yet more evidence that myelin protons can be directly imaged with UTE sequences on a clinical $3 \mathrm{~T}$ scanner: Bicomponent T2* analysis of native and deuterated ovine brain specimens. Magn Reson Med 2018;80:538-47.

16. Wilhelm MJ, Ong HH, Wehrli SL, Li C, Tsai PH, Hackney DB, Wehrli FW. Direct magnetic resonance detection of myelin and prospects for quantitative imaging of myelin density. Proc Natl Acad Sci 2012;109:9605-10.

17. Horch RA, Gore JC, Does MD. Origins of the ultrashortT21H NMR signals in myelinated nerve: A direct measure of myelin content? Magn Reson Med 2011;66:24-31.

18. Du J, Ma G, Li S, Carl M, Szeverenyi NM, VandenBerg S, Corey-Bloom J, Bydder GM. Ultrashort echo time (UTE) magnetic resonance imaging of the short $\mathrm{T} 2$ components in white matter of the brain using a clinical $3 \mathrm{~T}$ scanner. Neuroimage 2014;87:32-41.

19. Sheth V, Shao H, Chen J, Vandenberg S, Corey-Bloom J, Bydder GM, Du J. Magnetic resonance imaging of myelin using ultrashort Echo time (UTE) pulse sequences: Phantom, specimen, volunteer and multiple sclerosis patient studies. Neuroimage 2016;136:37-44.

20. Sheth VR, Fan S, He Q, Ma Y, Annese J, Switzer R, Corey-Bloom J, Bydder GM, Du J. Inversion recovery ultrashort echo time magnetic resonance imaging: A 
method for simultaneous direct detection of myelin and high signal demonstration of iron deposition in the brain A feasibility study. Magn Reson Imaging 2017;38:87-94.

21. Waldman A, Rees JH, Brock CS, Robson MD, Gatehouse PD, Bydder GM. MRI of the brain with ultra-short echotime pulse sequences. Neuroradiology 2003;45:887-92.

22. Seifert AC, Li C, Wilhelm MJ, Wehrli SL, Wehrli FW. Towards quantification of myelin by solid-state MRI of the lipid matrix protons. Neuroimage 2017;163:358-67.

23. Emid S, Creyghton JHN. High resolution NMR imaging in solids. Phys B+C 1985;128:81-3.

24. Jang H, Lu X, Carl M, Searleman AC, Jerban S, Ma Y, von Drygalski A, Chang EY, Du J. True phase quantitative susceptibility mapping using continuous single-point imaging: a feasibility study. Magn Reson Med 2019;81:1907-14.

25. Idiyatullin D, Corum C, Park JY, Garwood M. Fast and quiet MRI using a swept radiofrequency. J Magn Reson 2006;181:342-9.

26. Idiyatullin D, Corum CA, Garwood M. Multi-BandSWIFT. J Magn Reson 2015;251:19-25.

27. Zhang J, Idiyatullin D, Corum CA, Kobayashi N, Garwood M. Gradient-modulated SWIFT. Magn Reson Med 2016;75:537-46.

28. Hafner S. Fast imaging in liquids and solids with the Backprojection Low Angle ShoT (BLAST) technique. Magn Reson Imaging 1994;12:1047-51.

29. Wiesinger F, Sacolick LI, Menini A, Kaushik SS, Ahn S, Veit-Haibach P, Delso G, Shanbhag DD. Zero TE MR bone imaging in the head. Magn Reson Med 2016;75:107-14.

30. Weiger M, Pruessmann KP, Bracher AK, Köhler S, Lehmann V, Wolfram U, Hennel F, Rasche V. Highresolution ZTE imaging of human teeth. NMR Biomed 2012;25:1144-51.

31. Hövener JB, Zwick S, Leupold J, Eisenbeiß AK, Scheifele C, Schellenberger F, Hennig J, Elverfeldt DV, Ludwig U. Dental MRI: Imaging of soft and solid components without ionizing radiation. J Magn Reson Imaging 2012;36:841-6.

32. Schieban K, Weiger M, Hennel F, Boss A, Pruessmann KP. ZTE imaging with enhanced flip angle using modulated excitation. Magn Reson Med 2015;74:684-93.

33. Grodzki DM, Jakob PM, Heismann B. Ultrashort echo time imaging using pointwise encoding time reduction with radial acquisition (PETRA). Magn Reson Med 2012;67:510-8.

34. Jang H, Wiens CN, McMillan AB. Ramped hybrid encoding for improved ultrashort echo time imaging. Magn Reson Med 2016;76:814-25.

35. Jang H, Liu F, Bradshaw T, McMillan AB. Rapid dualecho ramped hybrid encoding MR-based attenuation correction (dRHE-MRAC) for PET/MR. Magn Reson Med 2018;79:2912-22.

36. Jang H, Liu F, Zhao G, Bradshaw T, McMillan AB. Technical Note: Deep learning based MRAC using rapid ultrashort echo time imaging. Med Phys 2018;45:3697-704.

37. Juras V, Apprich S, Szomolanyi P, Bieri O, Deligianni X, Trattnig S. Bi-exponential T2* analysis of healthy and diseased Achilles tendons: an in vivo preliminary magnetic resonance study and correlation with clinical score. Eur Radiol 2013;23:2814-22.

38. Juras V, Apprich S, Zbýň Š, Zak L, Deligianni X, Szomolanyi P, Bieri O, Trattnig S. Quantitative MRI analysis of menisci using biexponential $\mathrm{T} 2$ * fitting with a variable echo time sequence. Magn Reson Med 2014;71:1015-23.

39. Lu X, Ma Y, Chang EY, He Q, Searleman A, von Drygalski A, Du J. Simultaneous quantitative susceptibility mapping (QSM) and R2* for high iron concentration quantification with $3 \mathrm{D}$ ultrashort echo time sequences: An echo dependence study. Magn Reson Med 2018;79:2315-22.

40. Chen J, Carl M, Ma Y, Shao H, Lu X, Chen B, Chang EY, $\mathrm{Wu} \mathrm{Z}, \mathrm{Du} \mathrm{J}$. Fast volumetric imaging of bound and pore water in cortical bone using three-dimensional ultrashortTE (UTE) and inversion recovery UTE sequences. NMR Biomed 2016;29:1373-1380.

41. Ma YJ, Chang EY, Carl M, Du J. Quantitative magnetization transfer ultrashort echo time imaging using a time-efficient 3D multispoke Cones sequence. Magn Reson Med 2018;79:692-700.

42. Ma YJ, Lu X, Carl M, Zhu Y, Szeverenyi NM, Bydder GM, Chang EY, Du J. Accurate T1 mapping of short T2 tissues using a three-dimensional ultrashort echo time cones actual flip angle imaging-variable repetition time (3D UTE-Cones AFI-VTR) method. Magn Reson Med 2018;80:598-608.

43. Ma YJ, Zhao W, Wan L, Guo T, Searleman A, Jang H, Chang EY, Du J. Whole knee joint $\mathrm{T} 1$ values measured in vivo at $3 \mathrm{~T}$ by combined $3 \mathrm{D}$ ultrashort echo time cones actual flip angle and variable flip angle methods. Magn Reson Med 2019;81:1634-44.

44. Jang H, Wei Z, Wu M, Ma Y, Chang EY, Corey-Bloom $\mathrm{J}, \mathrm{Du} \mathrm{J}$. Improved volumetric myelin imaging in human brain using $3 \mathrm{D}$ dual echo inversion recovery-prepared 
UTE with complex echo subtraction. Magn Reson Med 2020;83:1168-77.

45. Ma YJ, Searleman AC, Jang H, Wong J, Chang EY, CoreyBloom J, Bydder GM, Du J. Whole-Brain Myelin Imaging Using 3D Double-Echo Sliding Inversion Recovery Ultrashort Echo Time (DESIRE UTE) MRI. Radiology 2020;294:362-74.

46. Jang H, Ma Y, Searleman AC, Carl M, Corey-Bloom J, Chang EY, Du J. Inversion recovery UTE based volumetric myelin imaging in human brain using interleaved hybrid encoding. Magn Reson Med 2020;83:950-61.

47. Wu Y, Dai G, Ackerman JL, Hrovat MI, Glimcher MJ, Snyder BD, Nazarian A, Chesler DA. Water- and fatsuppressed proton projection MRI (WASPI) of rat femur bone. Magn Reson Med 2007;57:554-67.

48. de Lange EE, Mugler JP, Bertolina JA, Gay SB, Janus CL, Brookeman JR. Magnetization Prepared RApid Gradient-Echo (MP-RAGE) MR imaging of the liver: Comparison with spin-echo imaging. Magn Reson Imaging 1991;9:469-76.

49. Grodzki DM, Jakob PM, Heismann B. Correcting slice selectivity in hard pulse sequences. J Magn Reson 2012;214:61-7.

50. Cheng Li, Magland JF, Seifert AC, Wehrli FW. Correction of Excitation Profile in Zero Echo Time (ZTE) Imaging

Cite this article as: Jang H, Carl M, Ma Y, Searleman AC, Jerban S, Chang EY, Corey-Bloom J, Du J. Inversion recovery zero echo time (IR-ZTE) imaging for direct myelin detection in human brain: a feasibility study. Quant Imaging Med Surg 2020;10(5):895-906. doi: 10.21037/qims.2020.04.13
Using Quadratic Phase-Modulated RF Pulse Excitation and Iterative Reconstruction. IEEE Trans Med Imaging 2014;33:961-9.

51. Lee YH, Suh JS, Grodzki D. Ultrashort echo (UTE) versus pointwise encoding time reduction with radial acquisition (PETRA) sequences at 3 Tesla for knee meniscus: A comparative study. Magn Reson Imaging 2016;34:75-80.

52. Weiger M, Brunner DO, Dietrich BE, Müller CF, Pruessmann KP. ZTE imaging in humans. Magn Reson Med 2013;70:328-32.

53. Du J, Bydder M, Takahashi A, Shimakawa A. Ultrashort TE (UTE) Imaging and T2 Quantification of Short T2 Components in Brain White Matter. In: Proceedings of Joint Annual Meeting ISMRM-ESMRMB. Berlin, Germany; 2007:3368.

54. Weiger M, Froidevaux R, Brunner DO, Rosler M, Pruessmann KP. Advances in Direct Myelin Imaging. In: Proceedings of the ISMRM 27th Annual Meeting \& Exhibition. Montreal, Canada; 2019:1118.

55. Lee HM, Weiger M, Giehr C, Froidevaux R, Brunner DO, Rösler MB, Pruessmann KP. Long-T2 -suppressed zero echo time imaging with weighted echo subtraction and gradient error correction. Magn Reson Med 2020;83:412-26. 